\title{
SPLINE ACTIVATED NEURAL NETWORK FOR CLASSIFYING CARDIAC ARRHYTHMIA
}

\author{
${ }^{1}$ Ganesh Kumar, R. and ${ }^{2}$ Y.S. Kumaraswamy \\ ${ }^{1}$ Department of Computer Science and Engineering, Sathyabama University, Chennai, India \\ ${ }^{2}$ Department of MCA-VTU, Dayananda Sagar College of Engineering, Bangalore, India
}

Received 2013-12-19; Revised 2014-03-18; Accepted 2014-04-09

\begin{abstract}
Electro Cardiogram's (ECG) biomedical signals characterizing cardiac anomalies are used for identifying cardiac arrhythmia. Irregular heartbeat-Arrhythmia-affects heart rate causing problems. Many methods, trying to simplify arrhythmia monitoring through automated detection, were developed over the years. ECG classification for arrhythmia is investigated in this paper based on soft computing techniques. RR interval are extracted from time series of the ECG and used as feature for arrhythmia classification. Frequency domain extracted features are classified using Radial Basis Function (RBF) and proposed Spline Activated-Feed Forward Neural Network (SAFFNN). Experiments were conducted with the Massachusetts Institute of Technology-Boston's Beth Israel Hospital (MIT-BIH) arrhythmia database for evaluating the proposed methods.
\end{abstract}

Keywords: Multilayer Perceptron, Feed Forward Neural Network, RR Interval, Arrhythmia Classification, ECG

\section{INTRODUCTION}

Arrhythmia is diagnosed through an Electro Cardiogram (ECG) procedure as abrupt/abnormal ECG beats represent arrhythmia. Diagnosis is based on long term data through an ECG recorder like the Holter recorder. Also, as remote and mobile healthcare systems using ECG recorders are increasing, it highlights the importance of an automatic arrhythmia classification algorithm. ECG is a much researched biomedical signal characterizing cardiac anomalies (Algazi and Meurin, 2000). Normal heartbeat is made up of waves based on the heart's mechanical actions. P-wave signifies auricular depolarization and complex QRS represents ventricular depolarization before mechanical contraction. The $\mathrm{R}$ wave has great amplitude as ventricle mass is more than that of auricles. T-wave represents ventricular repolarization (Singh and Tiwari, 2006). Usually cardiac anomaly traces appear in one/more ECG wave related parameters.

Arrhythmia diagnosis classification is made through acquired feature vectors following feature extraction. Many automated arrhythmia detection methods were developed over decades to simplify monitoring (Raut and Dudul, 2008). Pre-processing removes noise and includes more processing for accurate feature extraction/classification. ECG noise components include baseline drift, power line interference and moving artifacts (Friesen et al., 1990). Many filtering method reports were published for removal of noise components while simultaneously preserving ECG morphology/processing. Feature extraction leads to feature vectors used for classification.

The usual ECG pattern classification methods are Statistica approaches (Ge et al., 2002; Liu et al., 2004), Fuzzy inference approaches (Ceylan and Ozbay, 2007), Self-organizing map (Hussain and Fatt, 2007) and Neural Network approaches (Jadhav et al., 2010; Atoui et al., 2004). Artificial Neural Network (ANN) with universal approximation is used for classification (Mojarad et al., 2011). ANN Radial Basis Functions algorithms are also used for classification (Pang, 2005; Blu and Unser, 2002; Yu and Chen, 2007). The difference is that RBFs are local models and not MLPs. In RBF structure, training is split into three independent stages to reduce complexity and to ensure practical online usage.

Corresponding Author: Ganesh Kumar, R., Research Scholar, Department of CSE, Sathyabama University, Chennai, India. 
RR-intervals are the intervals between successive QRS detection points. Poor signal quality and errors in automatically generated QRS detections, RR-interval sequences from both sets of QRS detection times have physiologically unreasonable times (De Chazal et al., 2003). This study suggests an ECG processing methods analysis for each arrhythmia classification algorithm: Pre-processing, feature extraction and classification for the development of a robust algorithm. This study uses DCT to pre-process data to locate RR interval, which are used as features. A proposed Spline Activated feed forward neural network and RBF classifiers, classify the features as Left Bundle Branch Block (LBBB), Right Bundle Branch Block (RBBB) and normal.

\subsection{Related Works}

A new cardiac arrhythmia disease classification method described by Shivajirao et al. (2011) implements Modular Neural Network (MNN) framework to categorize arrhythmia into normal/abnormal classes. Experiments were undertaken on a UCI arrhythmia dataset. A FPG-based ECG signal classification based on block-based NN and a parallel GA was proposed by Jewajinda and Chongstitvatana (2010). A new arrhythmia classification algorithm consisting of fast learning speed and extremely accurate using Principal Component Analysis (PCA), Extreme Learning Machine (ELM) and Morphology Filtering was proposed by Kim et al. (2009) which categorized six heartbeat types. Various techniques/transformations proposed in literature earlier to extract features from ECG signals were discussed by Karpagachelvi et al. (2010). It also provided a comparative study of methods proposed by researchers to extract ECG signal features. An ECG signal processing method with Quad Level Vector (QLV) for ECG holter system was proposed by Kim et al. (2010) which consists of compression and classification flows. The QLV was proposed for both flows to achieve improved performance with lowcomputation complexity. The proposed compression technique reduced overall processing cost by $45.3 \%$.

A new approach to classify heartbeat based on combining morphological and dynamic features was proposed by Ye et al. (2012). Wavelet transform and Independent Component Analysis (ICA) were applied y to each heartbeat separately for morphological features extraction. Additionally, dynamic features were provided by RR interval information computation. The new method was validated on the baseline MIT-BIH arrhythmia database yielding an overall accuracy (percentage of heartbeats correctly classified) of $99.3 \%$ in "class-oriented" evaluation and $86.4 \%$ accuracy in "subject-oriented" evaluation, which compared favorably with state-of-the-art results for automatic heartbeat classification.

A generic and patient-specific classification system for robust and accurate ECG heartbeat patterns detection was presented by Ince et al. (2009) which utilized morphological wavelet transform features, projected on a lower dimensional feature space using PCA and ECG data's temporal features. Classification experiments on a benchmark database proved the new system achieved better results than most state-of-the-art algorithms in detecting Ventricular Ectopic Beats (VEBs) and supraVEBs (SVEBs). The proposed system was highly generic due to its parameter-invariant nature and thus was applicable to any ECG dataset.

Three active learning strategies for ECG signals classification was presented by Pasolli and Melgani (2010). Starting from small/suboptimal training sets, these strategies selected additional beat samples from large unlabeled data sets. The results revealed that the new strategies were capable of choosing samples significant for classification. A wearable module and NN based activity classification algorithm for energy expenditure estimation was presented by Lin et al. (2012) which consists of procedures for data collection, preprocessing, activity classification, feature selection and Energy Expenditure Regression (EER) models construction using NN. Experimental results successfully validated the wearable sensor module's effectiveness and its NN based activity classification algorithm for energy expenditure estimation. Additionally, the results proved the superior performance of GRNN compared to RBFN.

A method for automatic heartbeats classification in ECG signals was proposed by De Lannoy et al. (2012). As this task has specific characteristics like time dependences between observations and strong class unbalance, a specific classifier was suggested and evaluated on MIT arrhythmia database's real ECG signals. This was a weighted variant of conditional random fields' classifier. Experiments revealed that the new method outperformed earlier heartbeat classification methods, especially for pathological heartbeats.

A new algorithm for ECG signal compression based on local extreme extraction, adaptive hysteretic filtering and Lempel-Ziv-Welch (LZW) coding was presented by Fira and Goras (2008). The algorithm was verified using eight most frequent normal and pathological cardiac beats types and a Multi-Layer Perceptron (MLP) NN 
trained with original cardiac patterns and tested on reconstructed ones. The possibility of using PCA for cardiac pattern classification was also investigated.

A simple ECG feature models based heart beat classifier selected to improve generalization capability was studied and validated by Llamedo and Martinez (2011). Classification and generalization were studied using available databases like MIT-BIH Arrhythmia, MIT-BIH Supraventricular Arrhythmia and St. Petersburg Institute of Cardiological Technics (INCART) databases. A floating feature selection algorithm obtained best performing and generalizing models in training and validation sets for various search configurations. To test generalization capability, performance was evaluated in INCART with results comparable to that from the test set. This classifier model has reduced features performing better than state-of-the-art methods. Results suggest better generalization capability. The European database for evaluation of automatic detection of ST segment was used by Lee et al. (2013), which comprises many steps: ECG signal loading, signal preprocessing, QRS complex and Rpeak, ST segment detection and other relation parameter measurements. The application displays analysis results. The ECG signal analysis provides clinical information for heart disease diagnosis. ECG signal includes P, QRS complex and T-wave. The waves correspond to fields induced by the cardiac surface's specific electric phenomenon. Of them, ischemia detection is achieved by analyzing the ST segment. Ischemia is a widely prevalent and most serious heart disease.

Cardiac health is predicted by the ECG and heart rate. The nonlinear dynamics of ECG signals was studied by Anuradha and Reddy (2008) for the characterization of arrhythmia. Statistical measures of the features usually differ from the normal heart beat. The non linear features such as Spectral entropy, Poincaré plot geometry, Largest Lyapunov exponent and Detrended fluctuation analysis were used for ECG classification. Each feature was represented as fuzzy variable and conditional statements were derived to represent the knowledge base. This fuzzy classifier gave the overall accuracy of $93.13 \%$. Asl et al. (2008) proposed SVM classifiers with actual and reduced features to classify the heart beat signals. The features were reduced by Generalized Discriminant Analysis (GDA). HRV signals from the MIT-BIH Arrhythmia database were used for the classification and all the six types of arrhythmia types were taken as the target classes.

\section{MATERIALS AND METHODS}

This study uses RR interval for arrhythmia identification. RR peaks are detected as follows:

- The moving signal average is computed using six records

- New signals got by deducting moving average from original signal

- ' $\mathrm{R}$ ' signal peak is found

- Peaks of P, Q, S and T are found through relative position

Peak amplitude is measured from ' $\mathrm{k}$ ' line which is given by:

$$
\mathrm{k}=\operatorname{Max}\left(\theta_{\mathrm{i}}, \mathrm{i}=1,2, \ldots, 11\right)+\mathrm{c}
$$

where, $\theta_{\mathrm{i}}$ is the greatest amplitude, type of heartbeat and ' $c$ ' is a constant.

Figure 1 shows the RR measured using MATLAB. Arrhythmia beat-by-beat classification is through a set of rules on RR-interval signal. Rules are provided by medical experts based on clinical procedures to detect arrhythmic events from RR-intervals (Tsipouras et al., 2005). Rules are used for classification of middle RR interval of a 3 RR-interval sliding window. Classification concerns second beat of middle RR-interval. Beats are classified in three categories:

- Normal sinus beats $(\mathrm{N})$ and two arrhythmic ones

- Left Bunch Bundle Block (LBBB)

- Right Bunch Bundle Block (RBBB)

It is assumed that a beat, not from one of the above arrhythmic categories is classified as normal. The algorithm starts with window ' $i$ ' comprising of RR1i, RR2i and RR3i intervals. The middle RR-interval (RR2i) is a priori normal and classified in category 1 . MIT-BIH Arrhythmia Database was the first available standard test material to evaluate arrhythmia detectors and was used for that purpose and also for cardiac dynamics basic research at about 500 sites globally from 1980. It took five years to complete the MIT-BIH Arrhythmia Database (Moody and Mark, 2001). Tools used to create the database were primitive by current standards. ECG recordings were made using Del Mar Avionics model 445 two-channel reel-to-reel Holter recorders and analog signals recreated for digitization used a Del Mar Avionics model 660 playback unit. More than half MIT-BIH Arrhythmia Database is available via PhysioNet, enabling students and others to use a major portion of such data for cost free studies. 


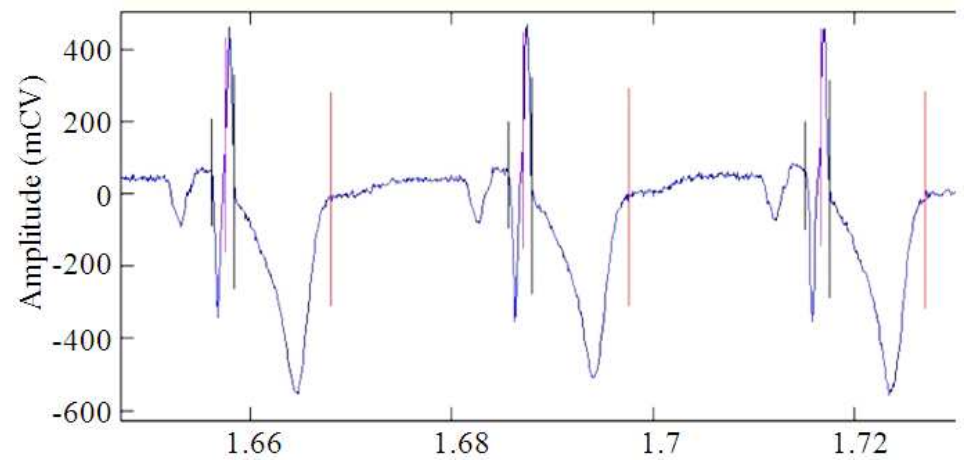

Fig. 1. RR wave shown in MATLAB

ECG datasets signals for training/testing are collected from MIT-BIH arrhythmia database (http://www.physionet.org/ physiobank/database/mitdb). Selected arrhythmias include LBBB, RBBB and Normal. Each ECG beat used is a matrix $(275 \times 1)$ for a ECG lead. Each ECG signal has five clear points (P, Q, R, S and T) for ECG interpretation.

Time series signal are converted into basis frequency components by Discrete Cosine Transform (DCT). Pre-processed data locates RR interval using Fast DCT (Che et al., 2011) of a list of 'n' real numbers $\mathrm{s}(\mathrm{x}), \mathrm{x}=0,1, . ., \mathrm{n}-1$, is list of length ' $\mathrm{n}$ ' given by:

$$
S(u)=\sqrt{2 / n} C(u) \sum_{x=0}^{n-1} s(x) \cos \frac{(2 x+1) u \pi}{2 n}
$$

where, $C(u)=2^{-1 / 2}$ for $\mathrm{u}=0$ or otherwise $\mathrm{C}(\mathrm{u})=1$.

Constant factors are chosen to ensure that basis vectors are orthogonal and normalized. The Inverse Discrete Cosine Transform (IDCT)

$$
S(x)=\sqrt{2 / n} \sum_{x=0}^{n-1} C(u) s(u) \cos \frac{(2 x+1) u \pi}{2 n}
$$

\subsection{Existing System}

\subsubsection{Feed-Forward Neural Networks (FNN)}

ANN is biologically inspired classification algorithms having an input node layer, one or more hidden layers and an output layer. Each layer node has a corresponding node in next layer, creating a stacking effect (Haykin, 2001). ANNs are versatile tools widely used to tackle issues. Feed-Forward Neural Networks (FNN) are popular among ANNs. These networks solve complex problems through modeling complex input- output relationships. The back-propagation algorithm is the workhorse for design of special class of layered feedforward networks known as Multi Layer Perceptrons (MLP). A popular layered feed-forward network is Radial-Basis Function (RBF) network with important universal approximation properties.

RBF networks are different from multilayer perceptrons in fundamental respects (Ileana et al., 2004):

- While RBF networks are local approximators, multilayer perceptrons are global approximators

- RBF networks have one hidden layer, while multilayer perceptrons have many hidden layers

- The RBF network's output layer is linear, while in a multilayer perceptron, it is linear/nonlinear

- The RBF network's hidden layer activation function computes Euclidean distance between input signal vector and the network's parameter vector, whereas a MLP's activation function computes inner product between input signal vector and pertinent synaptic weight vector

The feed-forward structure design leads to the minimizing generalization error, of learning time and of network dimension implies establishment of layer number, neuron number in every layer and interconnections between neurons. For now, there are no formal methods as an optimal choice for NN's dimensions. The number of layers choice is made knowing that a two layer network (one hidden layer) approximates most non-linear functions demanded by practice. A three layer network (two hidden layers) approximates any non-linear function. Hence, it ensures that a three layer network is enough for any problem (Ahmed and Natarajan, 1983). In reality use of large number of hidden layers is useful if each layer's neurons number is too big in a three layer approach. 


\subsection{Radial Basis Function (RBF)}

NN's have many neurons/computational unit layers all interconnected. Inputs fed on input layer are propagated through hidden layers to get an output. The latter is calculated through use of weights, bias and activation function (Lippman, 1987). The NN is trained between outputs and desired output which is the error. The following algorithms calculated various parameters in NN training. The input for a neuron is given:

$$
\mathrm{s}_{\mathrm{k}}=\sum_{\mathrm{j}} \mathrm{w}_{\mathrm{jk}} \mathrm{y}_{\mathrm{j}}+\theta_{\mathrm{k}}
$$

where, $S_{k}=$ The total or effective input for a unit ' $\mathrm{k}$ '

$\mathrm{W}_{\mathrm{jk}}=$ The weight of the connection

$\mathrm{Y}_{\mathrm{j}}=$ Current activation and

$\theta_{\mathrm{k}}=$ The bias

The function $\mathrm{A}_{\mathrm{f}}$ takes input and gets new activation value according to the following equation:

$$
y_{k}(t)=A_{f}\left(y_{k}(t-1) \cdot s_{k}(t-1)\right)
$$

Radial Basis Function (RBF) is a NN variant, better at interpolation and cluster modeling. RBF is embedded in a two layer neural network with radial activated function implemented in hidden layer (Broomhead and Lowe, 1998). Network outputs to inputs are fit to optimize network parameters during training which in turn is evaluated using Cost function and assumed to be square error. Gaussian activation function is used in pattern classification, given by (Wang and Jia, 2004):

$$
\begin{aligned}
& \phi_{j}(X)=\exp \left[-\left(X-\mu_{j}\right)^{T} \sum_{j}^{-1}\left(X-\mu_{j}\right)\right] \\
& \text { for } \mathrm{j}=1,2, \ldots, L,
\end{aligned}
$$

For $\mathrm{j}=1,2, \ldots, \mathrm{L}$, where ' $\mathrm{X}$ ' is input feature vector, ' $L$ ' the number of hidden units, ' $\mu_{\mathrm{j}}$ ' and ' $\Sigma_{\mathrm{j}}$ ' are means and covariance matrix of $j^{\text {th }}$ Gaussian function. Output layer implements a weighted hidden-unit outputs sum:

$$
\psi_{\mathrm{k}}(\mathrm{X})=\sum_{\mathrm{j}=1}^{\mathrm{L}} \lambda_{\mathrm{jk}} \varphi_{\mathrm{j}}(\mathrm{X})
$$

For $\mathrm{k}=1,2, \ldots, \mathrm{M}$.

RBF classifier's every hidden-layer node represents a class and constructs a hypersurface for it. These hypersurfaces are viewed as discriminant functions, with each hidden layer node producing a high value for the class it represents and low value for other classes. A
Gaussian RBF is a good choice for hidden layers, as it is a good similarity function (Bishop, 1995):

$$
\Phi(\overrightarrow{\mathrm{x}})=\exp \left(\frac{-\left\|\mathrm{x}_{\mathrm{i}}-\mu_{\mathrm{k}}\right\|^{2}}{2 \mathrm{~h} \cdot \sigma_{\mathrm{k}}{ }^{2}}\right)
$$

\subsection{Proposed System}

\subsubsection{Spline Activated Feed Forward Neural Network (SA-FFNN)}

The spline-based NN is built using Generalized Sigmoidal (GS) neuron, with adaptive parametric spline activation function (Guarnieri et al., 1995) which is easy to adapt and implement. It retains sigmoid's squashing property and smoothing characteristics. MLP constructed with spline activation functions are universal approximators with lowered structural complexity. Spline activation function (Vecci et al., 1998) reproduces total cubic spline shape along directions specified by $\mathrm{W}_{\mathrm{j}}$, where $\mathrm{j}=1,2, \ldots, n$ and defined by:

$$
\varphi\left(w_{j} x\right) \sum_{i=1}^{N} c_{i}\left|w_{j} x-\alpha_{i j}\right|^{3}
$$

$\mathrm{f}(\mathrm{x})$ can be written as:

$$
f(x)=\sum_{j=1}^{n} \mu_{j} \varphi_{j}\left(w_{j} x\right)
$$

$\mu_{\mathrm{j}}$ and $\mathrm{Wj}$ use back propagation, locating an optimal parameters and coordinates. Spline tracts are described by combining coefficients. Local spline basis functions controlled by 4 coefficients represent activation function. Catmull-Rom cubic spline is used and its $i^{\text {th }}$ tract is expressed as:

$$
\mathrm{F}_{\mathrm{i}}(\mathrm{u})=\left[\begin{array}{l}
\mathrm{F}_{\mathrm{x}, \mathrm{i}}(\mathrm{u}) \\
\mathrm{F}_{\mathrm{y}, \mathrm{i}}(\mathrm{u})
\end{array}\right]=\frac{1}{2}\left[\begin{array}{llll}
\mathrm{u}^{3} & \mathrm{u}^{2} & \mathrm{u} & 1
\end{array}\right]
$$

\section{RESULTS}

An energy extraction measure using Discrete Cosine Transform was used and RR interval extracted and used as a feature in this study. The extracted beats include 68 instances of left bunch bundle block, 30 instances of right bunch bundle block and 56 normal instances. The proposed SA-FF neural network was tested using 10 fold validation and compared with Radial Basis Function (RBF). The classification accuracy is given in Table 1 and Fig. 2. 
Table 2, Fig. 3 and 4 show the precision and recall of the classification methods used. For automated ECG arrhythmia classification system precision and recall play a crucial role in finding true positives and the proposed system improved both prevision and recall. It is observed that though the average precision of the proposed SAFNN is higher, it has lower precision for classifying normal instances. The proposed method improves the recall by $0.22 \%$ to $3.78 \%$ when compared to RBF.

\section{DISCUSSION}

The following Table 3 lists the accuracy of different classifiers for the classification of ECG Arrhythmia.

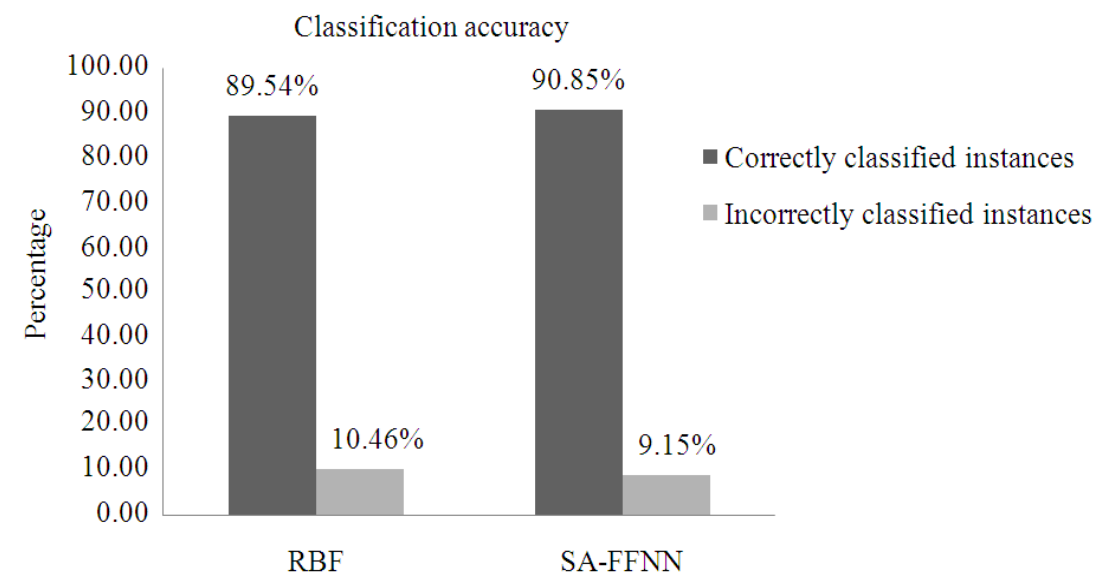

Fig. 2. Classification accuracy of various methods
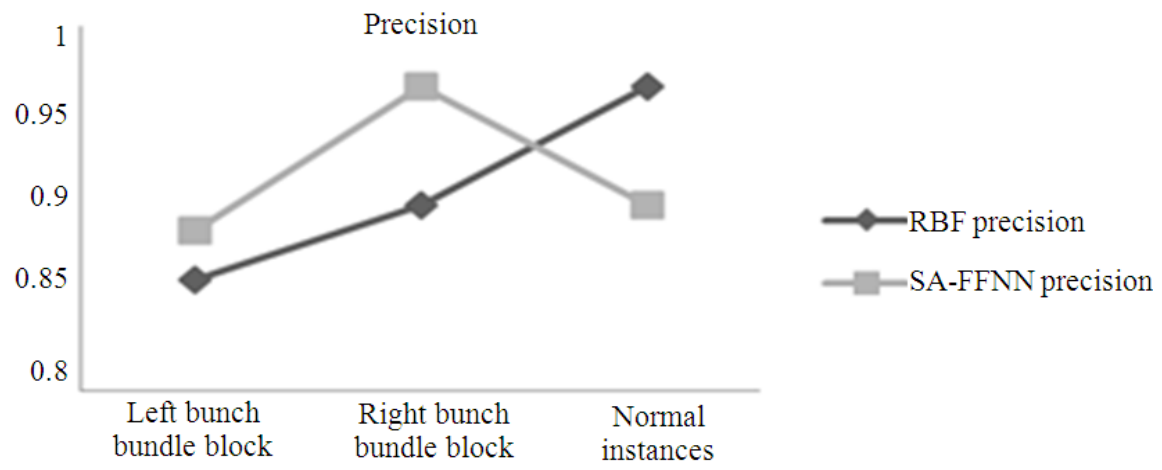

Fig. 3. Precision for various classification methods

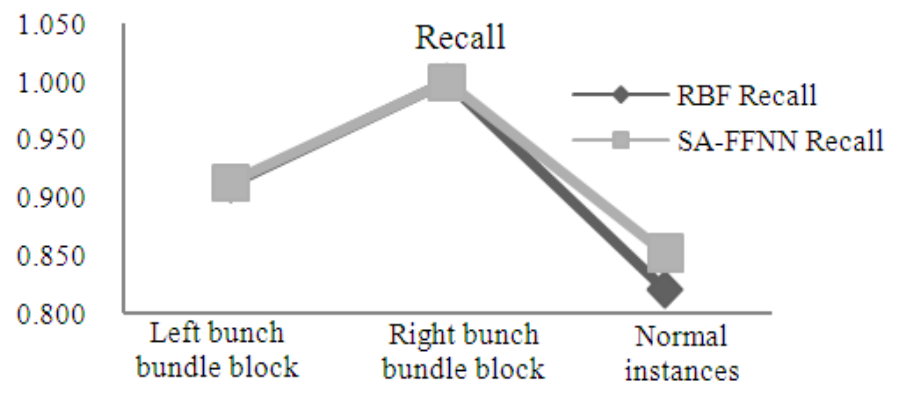

Fig. 4. Classification accuracy of various methods 
Table 1. Classification accuracy

\begin{tabular}{lllrr}
\hline Type of Acuracy & RBFF & $\%$ & SA-FFNN & $\%$ \\
\hline Correctly classified instances & 137 & 89.54 & 139 & 90.85 \\
Incorrectly classified instances & 16 & 10.46 & 14 & 9.15 \\
\hline
\end{tabular}

Table 2. Precision and recall

\begin{tabular}{|c|c|c|c|c|}
\hline \multirow[b]{2}{*}{ Type of Instances } & \multicolumn{2}{|l|}{ RBF } & \multicolumn{2}{|c|}{ SA-FFNN } \\
\hline & Precision & Recall & Precision & Recall \\
\hline Left bundle bunch block & 8.861 & 0.912 & 0.888 & 0.914 \\
\hline Right bundle bunch block & 0.902 & 1.000 & 0.967 & 1.000 \\
\hline Normal Instances & 0.967 & 0.821 & 0.902 & 0.852 \\
\hline
\end{tabular}

Table 3. Comparison of accuracy with various classifiers

\begin{tabular}{lll}
\hline Author & Classifier & Percentage of accuracy \\
\hline Asl et al. (2008) & SVM with reduced features & 99.16 \\
& SVM with original features & 98.49 \\
Anuradha and Reddy (2008) & Fuzzy Classifier & 93.13 \\
Proposed work & SA-FFNN classifier & 91.50 \\
\hline
\end{tabular}

\section{CONCLUSION}

Diagnosis systems are the base for several research domains in cardiology. Traditional classification methods include limitations in applications with neuronal techniques being considered promising algorithms to offset this. This study suggests an ECG Arrhythmia classification system using DCT for feature extraction in frequency domain. An improved Feed Forward NN based on spline activation function was suggested. The proposed Spline Activated Feed Forward NN (SAFFNN) improves classification accuracy by $1.31 \%$ when compared to RBF. Further work is needed in the field of $\mathrm{NN}$ to improve classification accuracy.

\section{REFERENCES}

Ahmed, N. and T. Natarajan, 1983. Discrete-Time Signals and Systems. 1st Edn., Reston Publishing Company, Inc. (A Prentice-Hall Company), Virginia. ISBN: 0835913759, pp: 398.

Algazi, M. and P. Meurin, 2000. L'électrocardiogramme: Guide Pratique. 1st Edn., Biopharma, Paris, pp: 248.

Anuradha, B. and V.C.V. Reddy, 2008. Cardiac arrhythmia classification using fuzzy classifiers. J. Theoretical Applied Inform. Technol., 4: 353-359.

Asl, B.M., S.K. Setarehdan and M. Mohebbi, 2008. Support vector machine-based arrhythmia classification using reduced features of heart rate variability signal. Artificial Intell. Med., 44: 51-64. DOI: 10.1016/j.artmed.2008.04.007
Atoui, H., J. Fayn and P. Rube, 2004. A neural network approach for patient-specific 12-Lead ECG synthesis in patient monitoring environments. Proceedings of the Computers in Cardiology, Sept. 19-22, IEEE Xplore Press, France, pp: 161-164. DOI: $10.1109 /$ CIC.2004.1442896

Bishop, C.M., 1995. Neural networks for pattern recognition. 1st Edn., Oxford University Press, pp:116-149.

Blu, T. and M. Unser, 2002. Wavelets, fractals and radial basis functions. IEEE Trans. Signal Proc., 50: 543553. DOI: $10.1109 / 78.984733$

Broomhead, D. and D. Lowe, 1998. Multivariable functional interpolation and adaptive networks. Complex Syst., 2: 321-355.

Ceylan, R. and Y. Ozbay, 2007. Comparison of FCM, PCA and WT techniques for classification ECG arrhythmias using artificial neural network. Expert Syst. Applic., 33: 286-295. DOI: 10.1016/j.eswa.2006.05.014

Che, Z.G., T.A. Chiang and Z.H. Che, 2011. Feedforward neural networks training: A comparison between genetic algorithm and back-propagation learning algorithm. Int. J. Innov. Comput. Inform. Control., 7: 5839-5850.

De Chazal, P., C. Heneghan, E. Sheridan, R. Reilly and P. Nolan et al., 2003. Automated processing of the single-lead electrocardiogram for the detection of obstructive sleep apnoea. IEEE Trans. BME, 50: 686-696. DOI: 10.1109/TBME.2003.812203 
De Lannoy, G., D. François, J. Delbeke and $M$. Verleysen, 2012. Weighted conditional random fields for supervised interpatient heartbeat classification. IEEE Trans. BME, 59: 241-247. DOI: 10.1109/TBME.2011.2171037

Fira, C.M. and L. Goras, 2008. An ECG signals compression method and its validation using neural networks. IEEE Trans. BME, 5: 1319-1326. DOI: 10.1109/TBME.2008.918465

Friesen, G.M., T.C. Jannett, M.A. Jadallah, S.L. Yates and S.R. Quint, 1990. A comparison of the noise sensitivity of nine QRS detection algorithms. IEEE Trans. BME, 37: 85-98. DOI: 10.1109/10.43620

Ge, D., N. Srinivasan and S. M. Krishnan, 2002. Cardiac arrhythmia classification using autoregressive modelling. Biomed. Eng. Online, 1: 5-5. DOI: 10.1186/1475-925X-1-5

Guarnieri, S., F. Piazza and A. Uncini, 1995. Multilayer neural networks with adaptive spline-based activation Functions. Proceedings of the International Neural Network Society Annu. Meet, (SAM' 95), Washington, DC., pp: I695-I699.

Haykin, S., 2001. Feed Forward Neural Networks: An Introduction. In: Nonlinear Dynamical Systems: Feedforward Neural Network Perspectives, Sandberg, I.W. (Ed.), John Wiley and Sons, New York, ISBN-10: 0471349119, pp: 1-16.

Hussain, H. and L.L. Fatt, 2007. Efficient ECG signal classification using sparsely connected radial basis function neural network. Proceeding of the 6th WSEAS International Conference on Circuits, Systems, Electronics, Control and Signal Processing, Dec. 29-31, Cairo, Egypt, pp: 412-416.

Ileana, I., C. Rotar and A. Incze, 2004. The optimization of feed forward neural networks structure using genetic Algorithms. Proceedings of the International Conference on Theory and Applications of Mathematics and Informatics, (AMI' 04), Greece, pp: 223-234.

Ince, T., S. Kiranyaz and M. Gabbouj, 2009. A generic and robust system for automated patient-specific classification of ECG signals, IEEE Trans. BME, 56: 1415-1426. DOI: 10.1109/TBME.2009.2013934

Jadhav, S.M., S.L. Nalbalwar and A.A. Ghatol, 2010. Arrhythmia disease classification using artificial neural network model. Proceedings of the IEEE International Conference on Computational Intelligence and Computing Research, Dec. 28-29, IEEE Xplore Press, India, pp: 1-4. DOI: 10.1109/ICCIC.2010.5705854
Jewajinda, Y. and P. Chongstitvatana, 2010. FPGAbased online-learning using parallel genetic algorithm and neural network for ECG signal classification. Proceedings of the International Conference on Electrical Engineering/Electronics Computer Telecommunications and Information Technology, May, 19-21, IEEE Xplore Press, Chaing Mai., pp: 1050-1054.

Karpagachelvi, S., M. Arthanari and M. Sivakumar, 2010. ECG feature extraction techniques-a survey approach. Int. J. Comput. Sci. Inform. Security, 8: 76-80.

Kim, H., R.F. Yazicioglu, P. Merken, C. Van Hoof and H.G. Yoo, 2010. ECG signal compression and classification algorithm with quad level vector for ECG Holter system. IEEE Trans. Inf. Technol. Biomed., 14: 93-100. DOI: 10.1109/TITB.2009.2031638

Kim, J., H.S. Shin, K. Shin and M. Lee, 2009. Robust algorithm for arrhythmia classification in ECG using extreme learning machine. Biomed. Eng. Online, 8: 31-31. DOI: 10.1186/1475-925X-8-31

Lee, D.H., J.W. Park, J. Choi, A. Rabbi and R. FazelRezai, 2013. Automatic detection of electrocardiogram ST segment: Application in ischemic disease diagnosis. Int. J. Advan. Comput. Sci. Appli., 4: 150-155.

Lin, C., Y. Yang and J. Wang, 2012. A wearable sensor module with a neural-network-based activity classification algorithm for daily energy expenditure estimation. IEEE Trans. Inf. Technol. Biomed., 16: 991-998. DOI: 10.1109/TITB.2012.2206602

Lippman, R., 1987. An introduction to computing with neural nets. IEEE ASSP Magazine, 4: 4-22. DOI: 10.1109/MASSP.1987.1165576

Liu, J., C. Wei and Y. Chen, 2004. Analysis of ECG R waves using adaptive wavelets transforms with fuzzy-logic. Proceedings of the 3rd International Conference on Computational Electromagnetics and Its Applications, Nov. 1-4, IEEE Xplore Press, pp: 423-425. DOI: 10.1109/ICCEA.2004.1459382

Llamedo, M. and J.P. Martínez, 2011. Heartbeat classification using feature selection driven by database generalization criteria. IEEE Trans. Biomed. Eng., 58: 616-625. DOI: 10.1109/TBME.2010.2068048

Mojarad, S.A., S.S. Dlay, W.L. Woo and G.V. Sherbet, 2011. Cross validation evaluation for breast cancer prediction using multilayer perceptron neural networks. Am. J. Eng. Applied Sci., 4: 576-585. DOI: 10.3844/ajeassp.2011.576.585 
Moody, G.B. and R.G. Mark, 2001. The impact of the MIT-BIH arrhythmia database. Eng. Medi. Biol. Magazine IEEE, 20: 45-50. DOI: $10.1109 / 51.932724$

Pang, S., 2005. Credit scoring model based on radial basis function network. Proceedings of the IEEE International Workshop on VLSl Design and Video Tech. May, 28-30, IEEE Xplore Press, China, pp: 389-392.

DOI: 10.1109/IWVDVT.2005.1504632

Pasolli, E. and R. Melgani, 2010. Active learning methods for electrocardiographic signal classification. IEEE Trans. Inf. Technol. Biomed., 14: 1405-1416. DOI: 10.1109/TITB.2010.2048922

Raut, R.D. and S.V. Dudul, 2008. Arrhythmias classification with MLP neural network and statistical analysis. Proceedings of the 1st IEEE International Conference on Emerging Trends in Engineering and Technology, July, 16-18, IEEE Xplore Press, India, pp: 553-558. DOI: 10.1109/ICETET.2008.260

Shivajirao, M.J., L.N. Sanjay and A.G. Ashok, 2011. Modular neural network based arrhythmia classification system using ECG signal data. Int. J. Inf. Technol. Knowl. Manage., 4: 205-209.
Singh, B.N. and A.K. Tiwari, 2006. Optimal selection of wavelet basis function applied to ECG signal denoising. Digital Signal Proc., 16: 275-287. DOI: 10.1016/j.dsp.2005.12.003

Tsipouras, M.G., D.I. Fotiadis and D. Sideris, 2005. An arrhythmia classification system based on the RRinterval signal. Artificial Intell. Med., 33: 237-250. DOI: 10.1016/j.artmed.2004.03.007

Vecci, L., R. Piazza and A. Uncini, 1998. Learning and approximation capabilities of adaptive spline activation function neural networks. Neural Netw., 11: 259-270. DOI: 10.1016/S0893-6080(97)00118-4

Wang, Y., Y. Jia, C. Hu and M. Turk, 2004. Face recognition based on kernel radial basis function networks. Beijing Institute of Technology.

Ye, C., Kumar and M.T. Coimbra, 2012. Heartbeat classification using morphological and dynamic features of ECG signals, IEEE Trans. BME, 59: 2930-2941. DOI: 10.1109/TBME.2012.2213253

Yu, S.N. and Y.H. Chen, 2007. Electrocardiogram beat classification based on wavelet transformation and probabilistic neural network. Periodical J. Patt. Recog. Lett., 28: 1142-1150. DOI: 10.1016/j.patrec.2007.01.017 